\title{
The Psychology of Cows - Commentary Response
}

\author{
Kristin Allen $^{1}$ and Lori Marino ${ }^{2,3 *}$ \\ ${ }^{1}$ Florida State University, Tallahassee, FL, USA \\ ${ }^{2}$ The Someone Project \\ ${ }^{3}$ The Kimmela Center for Animal Advocacy \\ *Corresponding author (Email: marinolori@ outlook.com)
}

Citation - Allen, K., \& Marino, L. (2017). The psychology of cows-commentary response. Animal Behavior and Cognition, 4(4), 530-532. https://dx.doi.org/10.26451/abc.04.04.15.2017

Keywords - Cow, Bos taurus, Cattle, Cognition, Intelligence, Emotions, Personality, Social

We are grateful for the thoughtful commentaries on our targeted review paper: The Psychology of Cows. These responses added important knowledge, insights, and analyses that greatly extended the discussion that we attempted to seed on the social, emotional, and cognitive complexity of cows. This review paper was, as King noted in her response, an "invitation to dialogue" about scientific knowledge of cows as complex sentient beings. We agree with King that this undertaking perhaps has latent challenges due to the cultural context in which it is embedded. In particular, we concur with several of our commentators, that the ubiquity of consumption and economic use of cows and the powerful ideology surrounding it has long shaped empirical inquiry and reactions to it. We acknowledge that we are attempting to summarize the psychological complexity of individuals whose bodies are a source of significant palate pleasure and profit. Americans consumed 24.8 billion tons of beef in 2015 (USDA ERS, 2017), and the beef industry in the US alone is worth the retail equivalent of over 105 billion dollars per year (USDA ERS, 2017). We appreciate King's recognition that we presented a comprehensive analysis of the literature on cows and did not succumb to "cherry-picking" favorable results. More importantly King acknowledges the widespread objectification of cows and other farmed animals even among other scientists, and that objectification leads to inhumane treatment.

Andrews underscores the dearth of non-applied research by noting the need for additional research that relates to personhood and moral consideration for cows. For example, she noted that, missing from the current cow data, are investigations of moral agency, in the form of data on cooperation, obedience to authority, guilt behaviors, mutualism, reciprocity, and solidarity behaviors. Additional data, she notes, could also strengthen our understanding of self-awareness, rational problem-solving, and autonomy in cows. We fully agree. All of these areas are valuable for ethicists to evaluate moral consideration of cows utilizing her model of a personhood stereotype. But as we found in our review paper, experiments on cows have largely been related to maximizing production and profit, or, only in some cases, to improve welfare, a point also noted by Nawroth and Langbein.

We recognized early on that the existing literature on cow behavior and cognition is biased toward applied themes. But, rather than simply summarizing the applied data, we structured the paper to hold the space for questions beyond it. We were not attempting to introduce bias, but to make explicit the bias that already exists but is currently denied. Our review paper had several goals: to bring to the scientific discourse the available evidence on cow complexity, and to juxtapose it alongside questions for its ethical implications; to combine fragmented empirical data into a coherent whole that represents the 
lived experience of cows; and to remain within the important parameters of sound scientific evidence while acknowledging that scientific knowledge is embedded in a powerful sociocultural context.

Along this line, we thank Rollin for his invaluable sociohistorical contextualization of our research. As he notes, Western culture largely values and rewards scientists and non-scientists alike for the objectification of cows and other nonhuman animals. Likewise, it marginalizes those who do not ascribe to this objectification. As Rollin (p. 528) noted: "Many traditional dairy scientists denigrate such concern as 'sentimental, anthropomorphic nonsense'. " Indeed, as Rollin noted, this culture of denigration of those who suggest alternatives to objectification has an extensive history. We hope that our review provides a small step in shifting the scientific discourse away from this marginalization and toward a more open, rigorous dialogue.

We appreciate the points made by Nawroth and Langbein about the utility of cognitive research for increasing welfare for farmed animals. Our aim was to provide a scientific basis for not only increased welfare practices but for examining, in a more general sense, our perspective on the use of cows and other farmed animals for food and products. We are grateful that many authors like Nawroth and Langbein are using empirical findings to formulate best practices. We are in full agreement with these authors that basic knowledge becomes valuable when applied to real problems and setting.

Regarding Hill's comments, we do want to emphasize, at the onset, that we are appreciative of her critique, as our goal in writing this paper was to open up a candid discussion of our perspectives on nonhuman animals, in this case, cows. Hill's main criticism of our paper appears to be that we are not "objective" in our presentation of the literature and our conclusions. For instance, she criticizes our use of the term "objectified," in reference to cows, suggesting that it is inflammatory. We believe it is selfevident that any animal who is bred and slaughtered solely for use as a food and material commodity is, by definition, objectified, or turned into an object. This is not meant to be inflammatory, or to "...elicit a very strong negative emotional response," as Hill (p. 507) charges, but to acknowledge the factual reality of cow use in order to better understand how it shapes research questions, data, and interpretations of cow psychology. Hill (p. 507) also challenges our assertion that factory farmed animals are treated in a "distressful and unnatural" manner. Her support for this is that Canadian and European animal welfare laws have "filtered into" US practices. She fails to mention that farmed animals are exempt from most state anti-cruelty laws, and there are no federal laws protecting farmed animals. There are also state exemptions for most commonly accepted agricultural practices (Bauer, 2008; Favre, 2016; also see Steier \& Patel, 2017). We reaffirm that intense confinement that restricts movement, interference with motherchild attachment bonds, and removing individuals' body parts, such as testicles and horns, is both distressing and unnatural. We appreciate Hill's critique but suggest that "objectivity" must be applied in all directions.

Several scholars noted important extensions of our review. Padilla and McElligott explicated the role of vocal communication in cows. They emphasized that vocal communication plays a central role in individual recognition and coordination of social behaviors. They noted that this is especially important in the mother - child bond, playing a critical role in the development of the appropriate behavioral repertoire as calves mature. Our paper and conclusions would be strengthened considerably by the material Padilla and McElligott discussed.

We also appreciate the points made by Mitchell and Makecha with regard to interpretation of data. We believe a rigorous discussion of potential interpretations of the empirical data are valuable for moving forward our understanding of cow psychology. In terms of the findings of Jensen, Duve, and Weary (2015) we stand corrected. However, it is also the case that pair-housing does work along with enhanced feeding to motivate play. Likewise, we are thankful to them for pointing out that our statement about cow play did not include pretend play and we agree that evidence of this kind of play in cows would be compelling support for self-awareness.

Fraser et al. emphasized that narrative data would serve as a valuable contribution to the body of scientific understanding of cows, and we concur. In particular, descriptive observations of how individual cows interact with other individuals, navigate their environments, interact with specific humans, how this changes over time, etc., provides valuable information regarding the psychology of cows that would be 
inaccessible using only reductionist approaches and experimental conditions. This is an important part of the data on cow psychology that is so greatly needed. We agree that some of these observational data might come from cattle ranchers, particularly if it is balanced with the understanding of the potential for inherent biases of these reports. This is because, as Fraser et al. noted, "Recognizing cattle as complex beings creates moral conflict" for farmers and ranchers (p. 504). Scientific evidence has long suggested that moral conflicts and the associated cognitive dissonance can lead to highly skewed interpretations of events that often serve a self-preservation role. We must seek ways to minimize bias in cow research and other nonhuman animal research. For this reason, narrative data from ranchers should be balanced with narratives from cow caretakers in other domains, such as in sanctuaries.

While we appreciate the suggestions made by Fraser et al. to incorporate more narrative description into our study of cows, we respectfully disagree with their assertion that we merely "stereotyped" farmers and ranchers by noting the indisputable fact that cows are treated as commodities by them. Indeed, cow bodies are sold for profit. This is the very definition of a commodity and of being treated as a commodity. Further, the failure of many in this industry to address even the most basic needs related to physical pain control and emotional distress by severing of their most natural, powerful, and well-documented affective maternal-child bonds for profit is exemplary of their exploitation as commodities in a profit-driven system. King provided a robust summary of this exploitation in her commentary. We therefore reaffirm our stance that cows are viewed and treated as commodities by the owners of production within the capitalist economy, and we assert that any efforts to dispute this provides further evidence of the ideology created by those with economic and political power. The most objective scientific inquiry will have the integrity to acknowledge and account for, rather than simply reproduce, this system of power.

Overall, we find the widely varied range of responses from so many esteemed scholars to be an indication of the importance of continuing this conversation. From those charging of anthropomorphic overreach, to those stating that our interpretations had been known colloquially for centuries, we believe there is much more to know about cow psychology. Importantly, we argue that a false pretense of scientific objectivity does a disservice to the cow literature.

We look forward to continuing this important discussion with these scholars and others who might be interested in this important issue. Acknowledging that cultural context shapes research questions, data, and interpretation, is an important step in reaching true scientific objectivity.

\section{References}

Bauer, G. (2008). Farm sanctuary: Changing hearts and minds about animals and food. New York, NY: Simon \& Shuster.

Favre, D. (2016). An international treaty for animal welfare. In D. Cao \& S. White (Eds.), Animal law and welfare: International perspectives (pp. 97-108). Heidelberg: Springer.

Jensen, M. B., Duve, L. R., \& Weary, D. M. (2015). Pair housing and enhanced milk allowance increase play behavior and improve performance in dairy calves. Journal of Dairy Science, 98, 2568-2575.

Steier, G., \& Patel, K. K. (Eds.) (2017). International farm animal, wildlife, and food safety law. Switzerland: Springer.

US Department of Agriculture, Environmental Research Service (2017). Retrieved from https://www.ers.usda.gov/topics/animal-products/cattle-beef/statistics-information.aspx. 